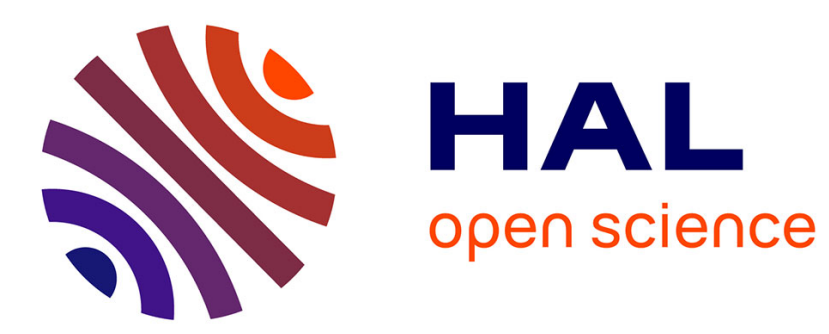

\title{
Transition from edge-localized to center-localized power deposition in helicon discharges \\ D. Curreli
}

\section{To cite this version:}

D. Curreli. Transition from edge-localized to center-localized power deposition in helicon discharges. European Physical Journal: Applied Physics, 2011, 56 (2), pp.24007. 10.1051/epjap/2011110159 . hal-00746216

\section{HAL Id: hal-00746216 https://hal.science/hal-00746216}

Submitted on 28 Oct 2012

HAL is a multi-disciplinary open access archive for the deposit and dissemination of scientific research documents, whether they are published or not. The documents may come from teaching and research institutions in France or abroad, or from public or private research centers.
L'archive ouverte pluridisciplinaire HAL, est destinée au dépôt et à la diffusion de documents scientifiques de niveau recherche, publiés ou non, émanant des établissements d'enseignement et de recherche français ou étrangers, des laboratoires publics ou privés. 


\title{
Transition from edge-localized to center-localized power deposition in helicon discharges
}

\author{
Davide Curreli ${ }^{1}$ \\ University of Padova, CISAS "G. Colombo", via Venezia 15, 35131, Padova, Italy \\ Received: date / Revised version: date
}

\begin{abstract}
In radiofrequency $(\mathrm{RF})$ helicon discharges the electromagnetic power is transferred from the $\mathrm{RF}$ field irradiated by the antenna to the plasma medium by means of plasma-wave coupling of the electromagnetic wave with the electrons. For the common industrial frequencies of tens of $\mathrm{MHz}$, and for typical pressures of few Pascals, the power deposition occurs mostly at the edge of the discharge. In these conditions, ionization and electron heating occurs in a layer close to the chamber walls, where a consistent fraction of the plasma is rapidly lost by diffusion toward the surface. The remaining fraction of plasma diffuses inward toward the center of the discharge, setting up a uniform and almost flat density profile, used in applications. A one-dimensional model considering both the plasma-wave coupling of the electrons with the RF wave and the macroscopic transport of ions and neutrals along the radial dimension of a cylindrical processing chamber has been derived, and used to evaluate the profiles at equilibrium. The model has been validated through Langmuir probe measurements in helicon processing chambers. The numerical model has then been used to study the power-coupling behavior of the discharge when the pressure of the neutral gas is decreased. When the Knudsen number of the neutral gas approaches the unity and in conditions of slightly magnetized discharge, the power deposition shifts from being edge-localized to center-localized, thus reducing the particle fluxes toward the walls and increasing the efficiency of the coupling.
\end{abstract}

\section{Introduction}

Radiofrequency helicon discharges represents the most efficient way to produce cold plasmas in a stable and quiescent state. Thanks to the high efficiency of this kind of source, and to the relative simplicity of construction, they have been studied for industrial processing as well as for high-efficiency space propulsion applications. Their efficiency relies on the mode-coupling of two electron plasma waves: a slow quasi-electrostatic wave and a fast whistler wave, excited by the presence of a magnetostatic field. In cylindrical discharges, the slow wave is rapidly dumped in a region at the boundary of the discharge, and it's responsible of most of the power transfer from the electromagnetic field radiated by the antenna to the plasma medium. The resulting absorption is highly spatially non-uniform, occurring mostly at the edge of the plasma volume, where diffusive losses toward the boundaries are not negligible.

The coupling efficiency of helicon sources can even be increased if the deposition of power could happen at the center of the discharge, in a region far from the walls. In order to assess this possibility, a consistent model of the discharge has been developed, taking into account not only the plasma-wave coupling, but also the macroscopic transport of species at equilibrium. As a result, the physical conditions for the transition from edge-localized to

\footnotetext{
Send offprint requests to: davide.curreli@unipd.it
}

center-localized power deposition has been assessed and discussed.

In the present work the model of the discharge has been reported (Sec. 2), considering the two problems of plasmawave coupling and macroscopic transport. The model allows a consistent evaluation of the equilibrium state of the discharge. The conditions for the transition to the centered deposition have been presented in Sec. 3, where the numerical results are reported and discussed.

\section{Discharge modeling}

\subsection{Plasma-wave coupling}

The analytical solution for a uniform plasma in cylindrical domains was obtained for the first time by Klozenberg in 1965 [1], and then generalized to the case of non-uniform plasmas by Chen and Arnush in the years 1997-2000 [2], [3], [4]. Accordingly to these models, the relevant behavior of helicons is obtained using a fluid model for electrons, satisfying the following set of equations:

$m\left[\frac{\partial \boldsymbol{v}}{\partial t}+(\boldsymbol{v} \cdot \nabla) \boldsymbol{v}\right]=-e\left(\boldsymbol{E}+\boldsymbol{v} \times \boldsymbol{B}_{0}\right)+\frac{\nabla \cdot \pi}{n_{0}}+\frac{\nabla p}{n_{0}}-m \nu \boldsymbol{v}$ 
together with the Maxwell equations in frequency domain:

$$
\begin{aligned}
\nabla \cdot \boldsymbol{B} & =0 \\
\nabla \times \boldsymbol{E} & =i \omega \boldsymbol{B} \\
\nabla \times \boldsymbol{B} & =\mu_{0} \boldsymbol{j}-i \omega \epsilon_{0} \boldsymbol{E}
\end{aligned}
$$

This system of equations can be simplified to a handling form after simple considerations on the more relevant terms, leading the fundamental helicon double-rotor equation:

$$
\delta \nabla \times \nabla \times \boldsymbol{B}-k \nabla \times \boldsymbol{B}+k_{w}^{2} \boldsymbol{B}=0
$$

where $k_{w}^{2}=\delta k_{s}^{2}$ is the wave number of a whistler wave propagating in the free space, $k_{s}=\omega_{p} / c$ is the skin number, which is the ratio between the plasma frequency $\omega_{p}$ and the speed of light, and $\delta=\omega / \omega_{c e}+i \nu / \omega_{c e}$ is the ratio between the frequency and the cyclotron frequency, corrected with the collisional term. When the density is not uniform along the cylinder radius, the fundamental equation 5 is modified by the addition of a source term [3]. The characteristic equation associated to Eq. 5 gives the dispersion relation:

$$
\delta \beta^{2}-k \beta+k_{w}^{2}=0
$$

whose solutions evidence the two allowed coupled-modes of propagation: the Helicon mode $\beta_{1}$, which is a low radial mode with high radial wavelength, and the TrivelpieceGould (TG) quasi-electrostatic mode $\beta_{2}$. The slow TG mode is highly damped at the boundary of the discharge, and it is the responsible of most the power deposition.

When written in one-dimension along the discharge radius, and Fourier transforming along the other two spatial dimensions and in frequency, the plasma-wave coupling problem reduces from a system of partial differential equations to a system of ordinary equations, that can be solved using ordinary numerical techniques. A detailed description of the model derivation, together with the assumptions and the equations obtained, can be found in Ref. [4]; the model is implemented in the HELIC code.

The specific power deposited into the plasma at each point is given by the projection of the electric field on the plasma currents. Then the total power deposited by the RF field into the plasma is obtained by integrating over the desired volume:

$$
P=\frac{1}{2} \int_{V} \boldsymbol{E}^{*} \cdot \boldsymbol{J}_{p l} d V=\frac{\left|I_{0}\right|^{2}}{2}(R+i X)
$$

where $R$ is the plasma resistance, $X$ the plasma reactance, $I_{0}$ the antenna current, and the upper script * denotes the complex conjugate of the vector. The power is transferred by means of both collisional resistive heating and noncollisional heating (Landau damping). In usual conditions, most of the power is transferred by collisional phenomena thanks to the TG wave, absorbed in a thin layer at the edge of the plasma cylinder. Experimental evidence of the $\mathrm{TG}$ absorption at the edge is given in [5].

\subsection{Macroscopic transport}

The theory of plasma-wave coupling presented up to here describes how the fields excited by the antenna deposit power into the plasma for given arbitrary profiles of $n, n_{n}$ and $T_{e}$, that are plasma density, neutral density and electron temperature respectively. When the plasma density is non-uniform along the radius, the absorption is greatly altered with respect to the case of uniform density. However, the radial profiles at equilibrium have been obtained by solving an additional set of equations, that account for the macroscopic transport of ions, electrons and neutrals inside the plasma cylinder. At equilibrium the species will be in a configuration depending on how the plasma is forced by the wave, and how the ions, electrons and neutrals interact together. In the present section we will address this problem, that will be referred as the macroscopic transport problem. All the quantities, comprising the power deposition, have been taken into account locally along the radius, with energy deposition described by the theory of plasma-wave coupling.

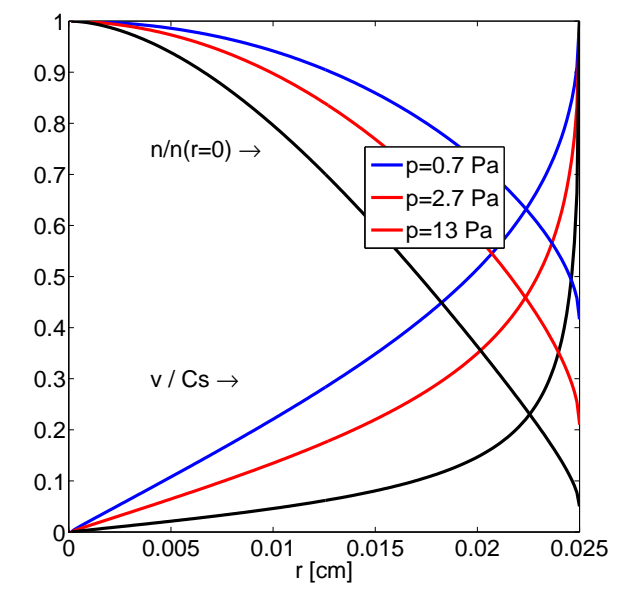

Fig. 1. Solutions of the radial transport of an Argon cylindrical discharge at three different pressures, showing the transition from the diffusive regime to the intermediate and to the freefall regime

We consider the radial dimension of a cylindrical plasma column, and we want describe the equilibrium conditions in the quasineutral region, where electron and ion densities are equal. At macroscopic level, stationary fluid equations describe the spatial evolution of the moments of the distribution functions, and they can proficiently be used as a first approximation for the evaluation of the discharge equilibrium. In stationary equilibrium conditions, the plasma generated at a given radius by ionization is equal to the plasma drifting to different radial stations,

$$
\nabla \cdot(n \boldsymbol{v})=n n_{n} P_{i}(r)
$$

where $n_{n}$ is the density of neutral gas, and the radiallydependent ionization probability $P_{i}(r) \equiv\left\langle\sigma v>_{i o n}(r)\right.$ is 
averaged over the distribution function, and varies sensitively with the electron temperature $T_{e}(r)$. The ion fluid is unmagnetized, and its equation of motion at equilibrium is:

$$
M n(\boldsymbol{v} \cdot \nabla) \boldsymbol{v}=e n \boldsymbol{E}-K_{B} T_{i} \nabla n-M n \boldsymbol{v} \nu_{i o}
$$

where $M$ is the ion mass, $n$ the quasineutral plasma density, $\boldsymbol{v}$ the ion fluid velocity, and $\nu_{i o}$ the charge-exchange collision frequency. The thermal component $K_{B} T_{i}$ of ion inertia can be neglected since the self-consistent electric field $\boldsymbol{E}=-\nabla \phi$ is scaled on the electron temperature $T_{e}>>T_{i}$. The equation of motion of magnetized electrons is given by

$m n \boldsymbol{v} \cdot \nabla \boldsymbol{v}=e n(\boldsymbol{E}+\boldsymbol{v} \times \boldsymbol{B})-K_{B} T_{e} \nabla n-m n \boldsymbol{v}\left(\nu_{e o}+\nu_{e i}\right)$

where both the electron-neutral and electron-ion collision frequencies are taken into account. When the short-circuit effect at the axial end-walls of the discharge is considered [7], electron dynamics is governed by a Boltzmann gradient relation in the form $d(\ln n) / d r=-d \eta / d r$, where the parameter $\eta$ is the quasineutral potential scaled on the local electron temperature $\eta=-e \phi / K_{B} T_{e}$.

Electrons are heated locally by the RF wave, and their temperature at equilibrium is given by the local energy balance between the deposited power and the losses

$$
W_{i}+W_{e}+W_{r}=P_{i n}
$$

where $W_{i, e}$ are the kinetic energies per unit time lost by ions and electrons leaving the plasma to the sidewalls and to the endplates, and $W_{r}$ is the power loss due to inelastic collisions, estimated using the Vahedi's curve [8].

Equations have been studied in one-dimensional cylindrical coordinates, assuming no variations along the other two dimensions, $\partial / \partial z=\partial / \partial \theta=0$. The system can be reduced to a system of ordinary differential equations, where the plasma drifts along the radial dimension varies according to the following non-linear equation:

$$
\frac{d u}{d \rho}=\frac{1}{1-u^{2}}\left[1+k u^{2}-\frac{u}{\rho}\right]
$$

where $u=v_{r} / C_{s}$ is the ratio between the radial (fluid) velocity and the ion acoustic velocity, $k \equiv 1+\left(P_{c} / P_{i}\right)$ is the ratio of ion-neutral and electron-neutral averaged collision probabilities, and $\rho \equiv\left(n_{n} P_{i} / C_{s}\right) r$ is a dimensionless rescaling of the spatial coordinate weighted on local plasma parameters. Eq.12 exhibits self-similar universal solutions and properties valid for both Helicon and ICPs discharges. The profiles of plasma potential $\phi$ and plasma density can then be explicitly obtained from Eq.12.

The dynamics of neutrals at equilibrium is taken into account by using a stationary diffusion equation forced by the plasma-neutral interaction, $D \nabla^{2} n_{n}=n_{n} n P_{i}$, where $D \equiv K_{B} T_{n} / M \nu_{n n}$ is the diffusion coefficient of isothermal neutrals.

Figure 1 shows an example of solution of the radial transport problem in the case of an Argon discharge with radius $r=2.5 \mathrm{~cm}$ at three different pressures. At high pressure, $\mathrm{p}=13 \mathrm{~Pa}$, the plasma solution approaches the classical Bessel diffusive solution [9]. At lower pressures, $\mathrm{p}=2.7 \mathrm{~Pa}$ and $\mathrm{p}=0.7 \mathrm{~Pa}$, the solution shifts continuously toward the Tonks-Langmuir free-fall solution.

\section{Transition to centered deposition}

Consistent calculations of the equilibrium conditions of helicon and ICP discharges [10] show that the power deposition occurs at the edge of the plasma cylinder for all the cases when the Knudsen number (ratio of the mean free path and the characteristic size) of the discharge is much smaller than one. In those cases, the absorption of RF power happens thanks to the collisional TrivelpieceGould mode, which is rapidly absorbed in a region near the external boundary of the plasma cylinder. When the pressure is reduced, the neutral gas approaches the Knudsen regime, and the collision rate is decreased as a consequence. In those conditions of low pressure, the absorption of the Trivelpiece-Gould mode at the boundary becomes less effective, due to the decreasing collision rate. By means of an opportune excitation of the coupled H-TG modes, the power deposition at low pressure shifts from being edge-localized and collisional in nature to centeredlocalized and wave-dominated. For a given pressure, the effect can be maximized in the range of low magnetic fields, where a peak in plasma resistance has also been observed in past works [6].

A cylindrical discharge with tube radius of $5 \mathrm{~cm}$ and length 2 meters, with a 16-cm-long half-helical antenna of $11 \mathrm{~cm}$ diameter, emitting at $f=13.56 \mathrm{MHz}$ have been considered, results are reported in Figs. 2, 3 and 4, showing the deposited power $P_{r}$ versus the radius, with the cylinder axis at $r=0$. In each graph $P_{r}$ is in the range $n=10^{17}-10^{18} \mathrm{~m}^{-3}$, for plasma processing applications.

The four figures reported in Fig. 2 show the case of a magnetized discharge with $B_{0}=500$ Gauss. In the diffusive regime at 1.4 Pascal and above, the power is absorbed at the boundary, where the TG mode is absorbed with peaks of $30 \mathrm{k} \Omega / \mathrm{m}^{2}$. Reducing the neutral pressure, $\mathrm{p}=0.8,0.27,0.13$ Pascal, decreases the TG peak, increases the skin depth of the deposition, but gives only a small increase in the centrally peaked deposition of $4 \%$ with respect to the dominant contribute at the boundary. This behavior can be explained by taking into account the radial extension of the two modes, as described by the dispersion relation Eq. 6. In fact, at 500 Gauss the two modes are well separated, with helicon radial modes in the range of $15-150 \mathrm{rad} / \mathrm{m}$ not fitting with the geometrical size of the discharge.

In Fig. 3 the magnetic field is reduced to 100 Gauss, and the low-pressure central peak of power absorption is seen to increase. For each case, when pressure is decreased, the absolute value of $P_{r}$ at the boundary decreases, and the skin depth of absorption increases due to the reduced collisionality. At 100 Gauss and pressures of 0.1 Pascals, the deposition is markedly centered, exhibiting a strongly coupled Helicon-TG mode with comparable wavelengths of the radial modes. 
The deposition at the center can be maximized when the magnetic field is further decreased to the range $40<$ $B_{0}<80$ Gauss, as showed in Fig. 4, where the centralpeaked deposition becomes dominant. The wave-heated nature of the deposition is revealed when $B_{0}$ is decreased below the evanescence conditions of the helicon branch (below 15 Gauss). Below this limit, the Helicon wave becomes an evanescent wave, and the peaked deposition disappears (the case $\mathrm{B}=10$ Gauss of Fig. 4). Here the discharge is shifting in the ICP mode, the H-TG coupling disappears, and the power deposition at the center falls of a factor 15 .

\section{Conclusions}

In usual conditions, helicon radiofrequency discharges absorb the electromagnetic power coming from the antenna in a region near the boundary of the plasma cylinder. Here we have shown that cylindrical helicon discharges offers the possibility to shift the RF power absorption from the boundary to the center. The transition occurs when the Knudsen number (ratio of the mean-free-path and the characteristic size) of the discharge approaches unity, and by exciting a strongly-coupled plasma wave with modal wavelengths comparable to the size of the discharge. The transition is made possible by the magnetized conditions of the plasma, and disappears when the magnetostatic field is decreased to zero and the discharge shifts to the ICP mode.

\section{Acknowledgments}

The author would like to express his deepest gratitude to prof. Em. Francis F. Chen (UCLA), for the theoretical and experimental collaboration during the development of this work.
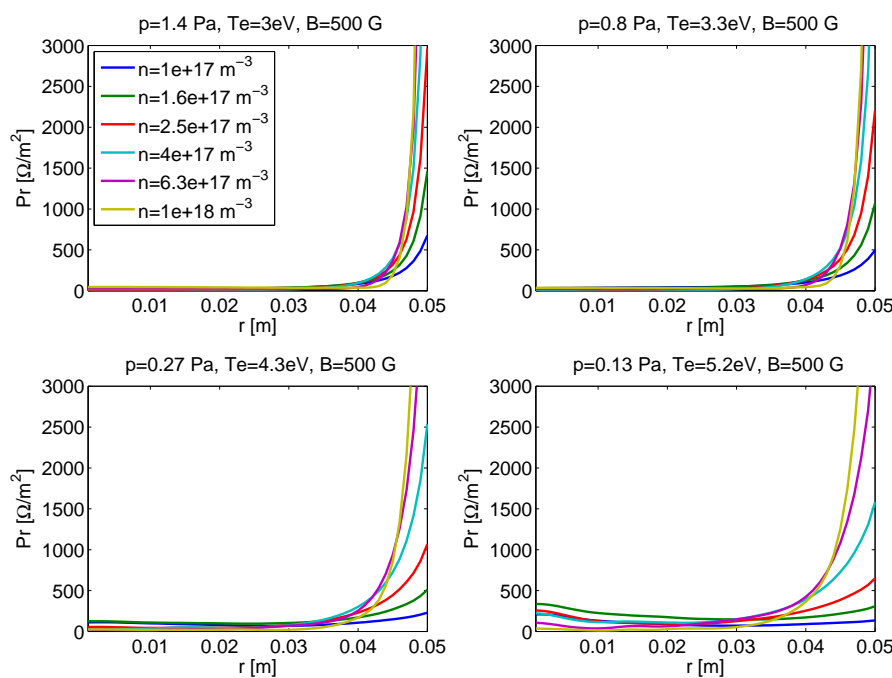

Fig. 2. Power deposition in discharges with magnetostatic field of 500 Gauss
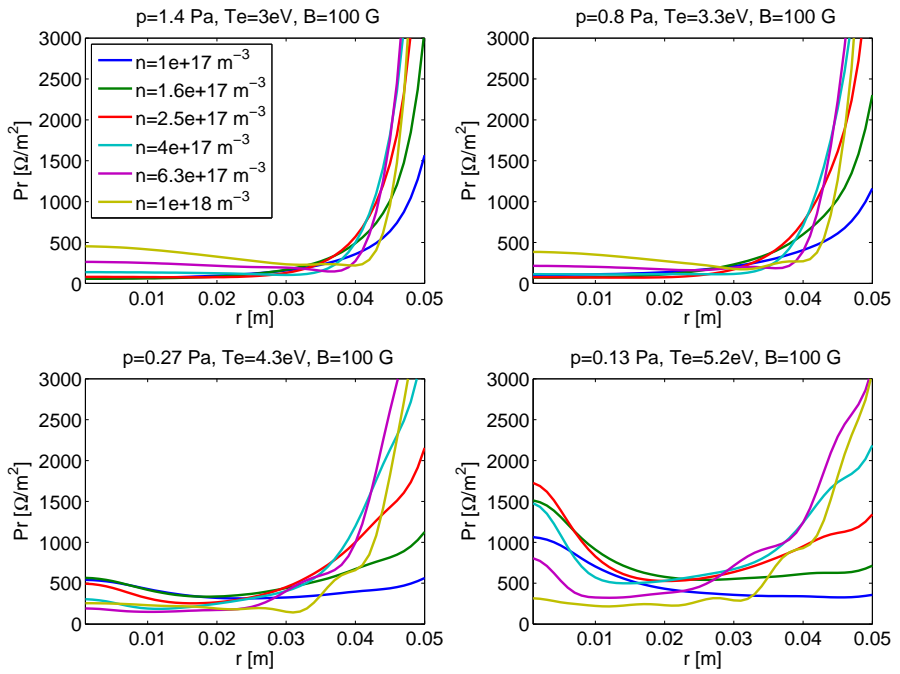

Fig. 3. Power deposition at 100 Gauss
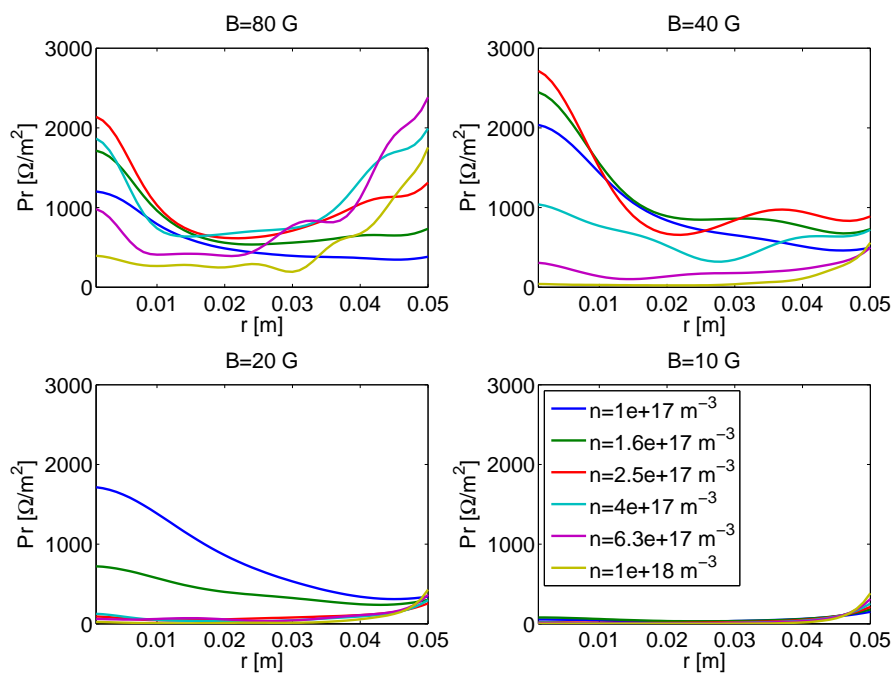

Fig. 4. Power deposition at low pressure for decreasing magnetic fields, for the cases $B=80,40,20$ and 10 Gauss; the gas pressure is $0.13 \mathrm{~Pa}$, electron temperature $T_{e}=5.2 \mathrm{eV}$

\section{References}

1. Klozenberg J. P., McNamara B. and Thonemann P. C., J. Fluid Mech. 21, (1965) 545.

2. F. F. Chen and D. Arnush, Phys. Plasmas 4, (1997) 3411.

3. D. Arnush and F. F. Chen, Phys. Plasmas 5, (1998) 1239.

4. D. Arnush, Phys. Plasmas 7, (2000) 3042.

5. D. D. Blackwell, T. G. Madziwa, D. Arnush and F. F. Chen, Physical Review Letters, 8814 (2002) 145002-1.

6. F. F. Chen, Phys. Plasmas 10, 6 (2003).

7. A. Simon, Phys. Rev. 98 (1955) 317.

8. V. Vahedi, Modeling and simulation of $r f$ discharges used for plasma processing, Thesis, University of California, Berkeley (1993).

9. F. F. Chen, Introd. to Plasma Physics and Controlled Fusion. Volume 1, Plasma physics, 2nd Ed., Springer (1984).

10. D. Curreli and F. F. Chen, A novel equilibrium theory of helicon discharges, 63rd GEC and 7th ICRP, (2010) DTP20. 